\title{
Robotic Management of Endometriosis: Discussion of Use, Criteria and Advantages: a Review of the Literature
}

\author{
Ioannis D. Gkegkes ${ }^{1, *}$, Christos lavazzo², George latrakis³, Paraskevi-Evangelia lavazzo ${ }^{4}$, Fani Pechlivani ${ }^{5}$, \\ Evangelia Antoniou ${ }^{5}$, Konstadia Bakalianou ${ }^{6}$
}

\begin{abstract}
Endometriosis is a very common benign condition affecting fertility and quality of life. Different methods, either definitive or fertility sparing are used for its management by using open, laparoscopic, and robotic techniques. This is a literature review presenting the role and the advantages of robotic surgery in endometriosis. Such a management is effective, safe, and feasible in hands of well-trained multidisciplinary teams even for severe cases of endometriosis.
\end{abstract}

\section{KEYWORDS}

da Vinci ${ }^{\odot}$ robot; endometriosis; treatment; advantages; criteria; quality of life

\section{AUTHOR AFFILIATIONS}

${ }^{1}$ Department of Surgery, Royal Devon and Exeter NHS Foundation Trust, Exeter, UK

2 Department of Gynaecological Oncology, The Christie Hospital, Manchester, UK

${ }^{3}$ Department of Obstetrics and Gynaecology, Technological Educational Institution of Athens, Athens, Greece

${ }^{4}$ Department of Paediatrics, General Hospital of Rethymno, Rethymno, Greece

${ }^{5}$ Department of Obstetrics and Gynaecology, Technological Educational Institution of Athens, Athens, Greece

${ }^{6}$ Department of Obstetrics and Gynecology, General Hospital "Agia Olga", Athens, Greece

* Corresponding author: 17C James House Residential Village, EX2 5DS, Bovemoors Lane, Exeter, UK; e-mail: ioannisgkegkes@gmail.com

Received: 4 July 2019

Accepted: 17 November 2019

Published online: 10 February 2020

Acta Medica (Hradec Králové) 2019; 62(4): 147-149

https://doi.org/10.14712/18059694.2020.3

(c) 2019 The Authors. This is an open-access article distributed under the terms of the Creative Commons Attribution License (http://creativecommons.org/licenses/by/4.0), which permits unrestricted use, distribution, and reproduction in any medium, provided the original author and source are credited. 


\section{INTRODUCTION}

Endometriosis occurs in 5-15\% of the general population and although a benign condition, sometimes it might require difficult surgical dissections as it could be locally infiltrative, invasive, and widely disseminated. The typical patient is nulliparous, infertile, and around 30-years-old. The most common sites of endometriosis intra-abdominally include adnexae (two out of three cases), pouch of Douglas, uterosacral and broad ligaments, uterovesical fold, ureters, bladder, appendix, rectosigmoid colon or caecum, and small bowel loops $(1,2)$.

The treatment options of endometriosis include medical (e.g. progestins, danazol, GnRH- analogues) or surgical options which could be classified as most definitive (including hysterectomy and bilateral salpingo-oophorectomy) or fertility-sparing with the aim to excise all peritoneal endometriotic implants and adhesions but preserve fertility (3-6). Laparoscopic or open techniques, depending on each surgeon's preference and experience, are offered as treatment options, while recently robotic procedures are also suggested.

The da Vinci ${ }^{\odot}$ surgical system received FDA approval in 2005. Robotic procedures have been introduced in order to improve surgical performance. Increased dexterity, greater range of motion, and better depth perception are the main advantages of robotic-assisted techniques (7). Its limitations include lack of tactile feedback and increased cost (8). Robotic procedures combine the advantages of open and laparoscopic procedures and are another alternative in the management of endometriosis.

The aim of this narrative review is to present the use, the criteria, and the advantages of robotic surgery in the treatment of endometriosis.

\section{DISCUSSION}

Different techniques including open, laparoscopic or robotic procedures can equally be used in the management of endometriosis (9-11). The patient is preoperatively assessed with imaging scans including ultrasound and MRI and she signs the informed consent when she is informed about the type of planned procedure and possible risks of it including infection, bleeding, and injury of adjacent organs. Multidisciplinary experts, including gynaecologists, urologists, and general surgeons, should cooperate in order to achieve the optimal outcome in the most severe cases.

Fertility-sparing techniques are used in order to destroy all endometriotic implants and remove all the possible adhesions. Removal and not lysis of them is preferred. Endometriomas larger than $3 \mathrm{~cm}$ are also excised either with cystectomy, or if that is not technically possible, with oophorectomy. If tubes are affected, salpingectomy is performed and IVF procedures are used for pregnancy achievement. If both adnexae are affected and bilateral salpingo-oophorectomy is essential, the uterus is preserved and donor eggs could be used for pregnancy. In robotic cases, all endometriotic implants are either excised or destroyed with scissors or diathermy. Segmental bowel resections, rectal shaving, and partial bladder resection are described in the literature (12). Ureteral endometriosis could be treated with partial ureterectomy and ureteroneocystostomy (13). Special care should be taken during the excision from small or large bowel and/or urinary tract to avoid any injuries. However, deep infiltrative endometriosis of the rectovaginal septum is one of the most severe types of endometriosis (14). For this reason, any hidden endometriosis should be completely excised to avoid developing deeper nodular lesions in the future (14). It was recently shown that infiltrating colorectal endometriosis could be safely and effectively treated robotically even by performing a rectosigmoidectomy if that is essential (1). In case of most definitive techniques, total or even modified radical hysterectomies plus bilateral salpingooophorectomies might be essential to treat the disease. Of course, as in open and laparoscopic techniques, preoperative use of $\mathrm{GnRH}$ analogues for three to six months can improve surgical success.

Robotic system preserves the advantages of conventional laparoscopy while it offers the possibility to the gynaecologist to dissect down and into the narrow pelvic floor. It is suggested that a diagnostic laparoscopy should be used in order to clarify the range of the disease in the upper abdomen before docking the robot in order to know exactly where the disease is. In robotic systems, the $\mathrm{CO}_{2}$ pressure required for exposure is often lower in correlation with traditional laparoscopy as result of the mechanical lift of the robot (15). Robotic procedures can be safely performed after taking into account the physiological changes of pneumoperitoneum and steep Trendelenburg position during a preoperative anaesthetic review (16). Robotics, also shares similar benefits of laparoscopy including smaller incisions (at most 10 to $12 \mathrm{~mm}$ ) (15). The three-dimensional stereoscopic vision by the use of binocular optics, the filtration of the tremor, and the less operator fatigue are some of the obvious advantages of such operations. The articulated instruments permit a wide range of motions while they increase the ability of the surgeon to work efficiently. All the above mentioned advantages can lead to more anatomical procedures. In addition, the $360^{\circ}$ motion of the robotic wrist permits the fine adhesiolysis and removal of any suspicious nodule, even if it is quite deep. More specifically, Patzkowsky et al., comparing robotic to laparoscopic treatment in over 500 patients, showed that age, body mass index, operative time, and estimated blood loss were not statistically different between the two procedures. Furthermore, robotic techniques could be easier used in larger uterus, cases with more severe adhesions, and stage III-IV endometriosis (17). However, the rates of urinary tract infections were higher in the robotic group (17). According to another study, including women treated with robotic-assisted laparoscopy for stage III and IV endometriosis, the median actual surgical time was 145 minutes (ranging from 67 to 325 minutes), while the median blood loss was $100 \mathrm{ml}$ (ranging from 20 to $400 \mathrm{ml}$ ) depending on the severity of the case and the experience of the surgeon (18). Another study group, showed that uterine weight higher than 250 grams and older age predispose to longer surgical time (19). In all those studies, the rates of conversion 
to open surgery and blood transfusion are minimal. So, the robotic assisted surgery also permits the realization of a key hole operation which can be interpreted into significantly less blood loss, less pain, shorter recovery time, as well as shorter hospital stay and better aesthetic result. Additionally, a shorter hospital stay and a quicker return to normal activity may mean less postoperative problems such as infection or pulmonary embolism. Last but not least, the use of robotic systems gives the opportunity of rapid acquisition of surgical skills required in order to perform laparoscopic surgery, while at the same time enable gynaecologists to reach at least as good clinical outcomes as conventional laparoscopy and within shorter operating times once they exceed the initial stage of the learning curve.

On the other hand, the high costs of use, the bulky machinery, and the need for staff training are the most important drawbacks in the utilization of robot in such operations. Of course, entry of new robotic systems in the market, as well as the use of the robot by different surgical teams, and in a high volume of patients could decrease the cost disadvantage. Short term complications include vaginal cuff abscess (18), ureterovaginal fistulas (20), and higher rates of urinary tract infection caused by extended use of Folley catheter for urinary retention (21). A rare case of rhabdomyolysis and compartment syndrome, after a 12-hour duration robotic operation, is also presented in the literature (22), showing the need for training and time managing of such operations. Although larger prospective studies as well as longer follow-up periods are necessary to clarify the long term outcomes including fertility results, pain, and quality of life, it seems that robotic management of even severe cases of endometriosis is an effective, feasible, and safe alternative in well-trained hands as it was also shown in a recent systematic review (23) and can be used without compromising the principles of open or laparoscopic operations.

\section{CONFLICT OF INTEREST}

No conflict of interest was declared by the authors.

\section{FINANCIAL DISCLOSURE}

The authors declared that this study has received no financial support.

\section{REFERENCES}

1. Neme RM, Schraibman V, Okazaki S, et al. Deep infiltrating colorectal endometriosis treated with robotic-assisted rectosigmoidectomy. JSLS 2013; 17(2): 227-34.

2. Gui B, Valentini AL, Ninivaggi V, Marino M, Iacobucci M, Bonomo L. Deep pelvic endometriosis: don't forget round ligaments. Review of anatomy, clinical characteristics, and MR imaging features. Abdom Imaging 2014; 39(3): 622-32.

3. Jacobson TZ. Potential cures for endometriosis. Ann N Y Acad Sci 2011; 1221: 70-4.

4. Grandi G, Xholli A, Napolitano A, Palma F, Cagnacci A. Pelvic pain and quality of life of women with endometriosis during quadriphasic estradiol valerate/dienogest oral contraceptive: a patient-preference prospective 24-week pilot study. Reprod Sci 2015; 22(5): 626-32.

5. Agarwal S, Fraser MA, Chen I, Singh SS. Dienogest for the treatment of deep endometriosis: case report and literature review. J Obstet Gynaecol Res 2015; 41(2): 309-13.

6. Yamaguti EM, Brito MB, Ferriani RA, Garcia AA, Rosa-e-Silva JC, Vieira CS. Comparison of the hemostatic effects of a levonorgestrel-releasing intrauterine system and leuprolide acetate in women with endometriosis: a randomized clinical trial. Thromb Res 2014; 134(6): 1193-7.

7. Iavazzo C, Gkegkes ID. Single-site port robotic-assisted hysterectomy: a systematic review. Arch Gynecol Obstet 2014; 289(4): 725-31.

8. Iavazzo C, Papadopoulou EK, Gkegkes ID. Cost assessment of robotics in gynecologic surgery: a systematic review. J Obstet Gynaecol Res 2014; 40(11): 2125-34.

9. Duffy JM, Arambage K, Correa FJ, et al. Laparoscopic surgery for endometriosis. Cochrane Database Syst Rev 2014; (4): CD011031.

10. Roman H. Endometriosis surgery and preservation of fertility, what surgeons should know. J Visc Surg 2018; 155 Suppl 1: S31-S36.

11. Nezhat C, Lewis M, Kotikela S, et al. Robotic versus standard laparoscopy for the treatment of endometriosis. Fertil Steril 2010; 94(7): 2758-60.

12. Siesto G, Ieda N, Rosati R, Vitobello D. Robotic surgery for deep endometriosis: a paradigm shift. Int J Med Robot 2014; 10(2): 140-6.

13. Frick AC, Barakat EE, Stein RJ, Mora M, Falcone T. Robotic-assisted laparoscopic management of ureteral endometriosis. JSLS 2011; 15(3):396-9.

14. Nezhat FR, Mahmoud MS. Allen masters peritoneal defect: a potential pathway to deep infiltrating rectovaginal endometriosis? J Minim Invasive Gynecol 2014; 21(3): 321-2.

15. Iavazzo C, Gkegkes ID. Port site metastases after robot-assisted surgery: a systematic review. Int J Med Robot 2013; 9(4): 423-7.

16. Gupta K, Mehta Y, Sarin Jolly A, Khanna S. Anaesthesia for robotic gynaecological surgery. Anaesth Intensive Care 2012; 40(4): 614-21.

17. Patzkowsky KE, As-Sanie S, Smorgick N, Song AH, Advincula AP. Perioperative outcomes of robotic versus laparoscopic hysterectomy for benign disease. JSLS 2013; 17(1): 100-6.

18. Bedaiwy MA, Rahman MY, Chapman M, et al. Robotic-assisted hysterectomy for the management of severe endometriosis: a retrospective review of short-term surgical outcomes. JSLS 2013; 17(1): 95-9.

19. Giep BN, Giep HN, Hubert HB. Comparison of minimally invasive surgical approaches for hysterectomy at a community hospital: robotic-assisted laparoscopic hysterectomy, laparoscopic-assisted vaginal hysterectomy and laparoscopic supracervical hysterectomy. J Robot Surg 2010 Sep; 4(3): 167-75.

20. McAchran SE, Paolone DR. The iatrogenic ureterovaginal fistula. Urology 2013; 81(6): e35.

21. Smorgick N, DeLancey J, Patzkowsky K, Advincula A, Song A, AsSanie S. Risk factors for postoperative urinary retention after laparoscopic and robotic hysterectomy for benign indications. Obstet Gynecol 2012; 120(3): 581-6.

22. Deras P, Amraoui J, Boutin C, Laporte S, Ripart J. [Rhabdomyolysis and compartment syndrome of two forearms after robotic assisted prolonged surgery]. Ann Fr Anesth Reanim 2010; 29(4): 301-3.

23. Carvalho L, Abrão MS, Deshpande A, Falcone T. Robotics as a new surgical minimally invasive approach to treatment of endometriosis: a systematic review. Int J Med Robot 2012; 8(2): 160-5. 